\section{Impact of the Laser on Dental Caries}

The characteristics of the laser beam which are of great interest and importance from the biomedical aspect are the ability to direct and to focus, intense energy density and some selectivity ${ }^{1}$. Since the laser beam is a light beam, it can be focused most accurately by using lens systems. Energy density is intense. In these experiments, the energy density was approximately 9,000 joules $/ \mathrm{cm}^{2}$ in the target area. Selectivity indicates that materials which are coloured absorb more of the incident beam of the lasers now available than uncoloured materials. In addition to these properties, of interest to the dentist is the fact that the duration of the impact is in the order of a millisecond.

Following a series of experiments of a study on impact of high-energy lasers, 300-joules exit energy, on bone, preliminary studies were done of the impact of the laser on teeth. These experiments were done with a pulsed ruby laser, 90-joules exit energy, beam width approximately $3 / 8$ in., with an energy density of the focused beam of approximately 9,000 joules $/ \mathrm{cm}^{2}$. Some experiments were done also with special glass rods which transmitted the laser beam directly into the cavities. The teeth used in these experiments were fastened to holders on the optical bench. Pre- and post-impact pictures were obtained with a standard colour photography set-up. The teeth after impact were observed under a stereobinocular microscope. In these studies, sections have been made of the teeth by John Gray and will be reported later.

The report will be concerned with the effects on areas of caries. Subsequent reports will give details of effects of drilling of the tooth, temperature of the root canal after impacts, effect on calculi, vaporization of amalgams and porcelains and also the development of techniques to permit direct impact on selected areas of teeth in patients. In addition, impacts have been done to attempt to make the surface of the teeth more resistant to infection.

The teeth were not hot on the outside, even after repeated impacts. The effect on the area of caries varied from small 2-mm deep holes to complete disappearance of the caries tissue (Fig. 1), with some whitening of the rim around. With the use of lasers with high repetition

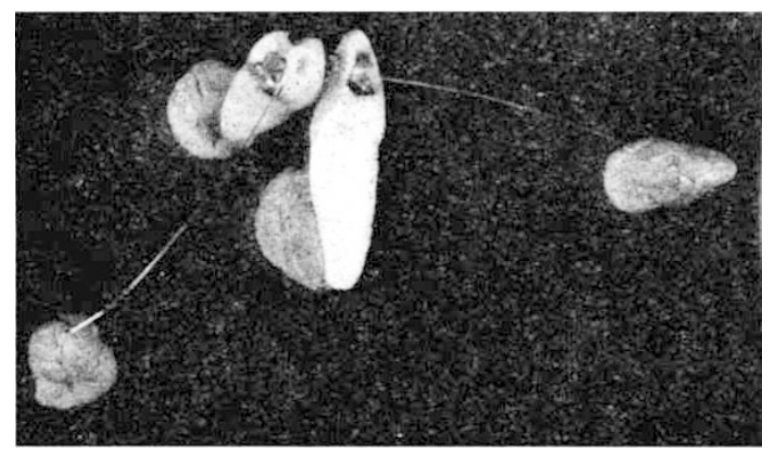

Fig. 1. Depth of impact on the buccal cusp of the lower bicu spid as compared with the impact on the mesial carious portion of the upperight central incisor. Impact from focused beam of pulsed ruby laser with exit energy of 90 joules. The increased penetration of the carious portion is due to the darker colour of this area

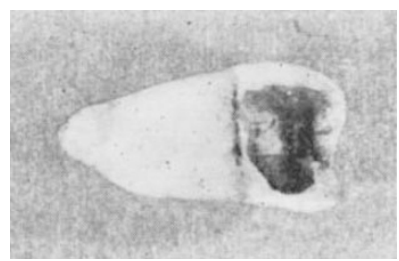

Fig. 2. Broad area of impact in a deep carious area by transmission of the laser beam through a special glass rod. The laser beam is from a pulsed ruby laser of 90 -joules exit energy rate of firing, the areas of destruction by focused beam will be greater. In several experiments, the unfocused boam of the laser was transmitted through special glass rods. Approximately 38 per cent of the energy was transmitted. These experiments were performed to show how the laser beam may be used in inaccessible areas on the tooth (Fig. 2). The unfocused beam with energy-levels as indicated produced broad but superficial areas of removal of the brownish carious material. Focused impacts produced deeper areas of removal of the infected material. In this series, no cultures were made pre- and post-impact, since the teeth specimens were not fresh. Thermistor readings were taken with large thermistors. Significant temperature rises were recorded. However, the response time of the thermistors was quite long and the experiments will be continued with faster thermistors.

Preliminary experiments indicate extensive destruction of carious areas of teeth by the unfocused and focused beams of the high-energy pulsed ruby laser. Areas of destruction were smaller but deeper with focused beams. These investigations indicate that the applications of the laser to dentistry should be examined in great detail, not only as regards basic studies, but also for the application for investigative clinical research.

This work was supported by a grant from the John A. Hartford Foundation.

\section{LEON GoLDMAN Peter Hornby Robert Meyer BERNARD GOLDMAN}

Laser Laboratory,

Children's Hospital Research Foundation, Cincinnati, Ohio.

${ }^{1}$ Goldman, Leon, Blaney, Donald J., Kindel, Daniel J., Richfield, Daniel, and Franke, Ernst K., Nature, 197, 912 (1963).

\section{IMMUNOLOGY}

\section{Production of SV-40 Complement-fixing Antigen}

MAYER et al. ${ }^{1}$ describe the preparation of complementfixing antigen of vacuolating agent $S V-40$ in monkey kidney cell cultures. Strain No. 776 of $S V-40$ used for the work described here was obtained from Dr. H. M. Meyer. In our attempts to prepare a satisfactory complement-fixing antigen, two types of cell cultures were used: primary African green monkey kidney (AGMK) and serially propagated $B S-C-1$ (ref. 1). These cultures were obtained from Microbiological Associates, Inc., and maintained at $34^{\circ} \mathrm{C}$ on Eagle's basal medium containing 2 per cent agamma ealf serum, $100 \mathrm{mg}$ of streptomycin per ml., 100 units of penicillin per $\mathrm{ml}$. and $2 \mathrm{mM}$ of glutamine per ml.

Following inoculation they were fed once a week until appearance of definite cytopathic effect. With small doses cytopathic effect appeared within 3-4 weeks. With larger inocula incubation time was shortened to 2-4 days. Tissue cultures were collected at the time of maximum cytopathic effect by freezing at $-70^{\circ} \mathrm{C}$. On thawing out, the cells were shaken off the glass and homogenized for $1 \mathrm{~min}$ at maximum speed in a Servall high-speed omnimixer. The resulting crude suspension was used as complement-fixing antigen. As antibody source, a $1 / 40$ dilution of pooled 'normal' monkey serum with an original complement-fixing titre of $1 / 128$ was used. At multiplicities greater than 10 , relatively little complementfixing antigen as measured in the 'microtitre' system² was obtained. The complement-fixing antigen yield increased significantly, however, at lower virus inputs (Table 1).

This observation was confirmed when 32-oz. flasks of AGMK and $B S-C-1$ cultures were inoculated with approximately $10-1,000 T C I D_{50}$ of $S V-40$ virus (Table 2). 